Case Report

\title{
A Dual Case of Peritonitis and Central Nervous System Infection Caused by Nutritionally Variant Streptococcal Species
}

\author{
Sussi Vivar, ${ }^{1}$ Jennifer E. Girotto, ${ }^{1,2}$ and Thomas S. Murray ${ }^{1,3}$ \\ ${ }^{1}$ Connecticut Children's Medical Center, Hartford, CT, USA \\ ${ }^{2}$ School of Pharmacy, University of Connecticut, Storrs, CT, USA \\ ${ }^{3}$ Frank H. Netter MD School of Medicine, Quinnipiac University, Hamden, CT, USA \\ Correspondence should be addressed to Thomas S. Murray; tmurray@connecticutchildrens.org
}

Received 25 July 2016; Accepted 4 January 2017; Published 23 January 2017

Academic Editor: Paola Di Carlo

Copyright (C) 2017 Sussi Vivar et al. This is an open access article distributed under the Creative Commons Attribution License, which permits unrestricted use, distribution, and reproduction in any medium, provided the original work is properly cited.

\begin{abstract}
Nutritional variant streptococci (NVS) are difficult to identify bacteria that can cause invasive infections such as endocarditis and meningitis. NVS as a cause of peritonitis has not been routinely described. This case of NVS as the etiology of peritonitis associated with previous neurosurgery and ventriculoperitoneal (VP) shunt revision demonstrates its potential role as a significant pathogen in patients with peritonitis and VP shunts. Therapy consists of vancomycin plus a second agent but since there are no standards for susceptibility testing, clinical response remains the standard for determining the efficacy of treatment. When there is central nervous system (CNS) involvement it is important to include drugs with appropriate CNS penetration.
\end{abstract}

\section{Introduction}

Nutritionally variant streptococci (NVS) comprise the Abiotrophia sp. and Granulicatella sp. and are normal residents of the oral cavity, gastrointestinal, and urogenital tracts $[1,2]$. Given that NVS are fastidious, grow slowly, and can be pleiomorphic on Gram stain, these bacteria may be misidentified [1]. NVS causes a variety of invasive infections, most commonly endocarditis as a result of bacteremia [2]. Other reported infections include osteomyelitis, otitis media, wound infections, septic arthritis, and pancreatic abscesses and infections of the CNS [2-4]. Here we describe a case of CNS shunt associated peritonitis where the cultures from the CNS grew pure NVS. While peritonitis is a well-known complication of VP shunt infections, this organism as a pathogen in significant abdominal infections in children is rarely reported.

\section{Case Presentation}

A 13-year-old girl with hydrocephalus managed with bilateral ventriculoperitoneal (VP) shunts presented to the Emergency Department (ED) with 24 hours of worsening generalized abdominal pain, headaches, dizziness, and lethargy without fever. She was born prematurely at 27 weeks of gestation resulting in developmental delay and her adoptive parents provided the history. Her parents reported she had decreased oral intake for four days prior to presentation with chills but no fever, emesis, or urinary symptoms. She had $20 \mathrm{VP}$ shunt revisions in her lifetime, the most recent being completed two months prior to presentation. Her additional past medical history was significant for epilepsy, chronic headaches, cerebral palsy, systemic lupus erythematosus, constipation, and gastroesophageal reflux disease. Her medication list included Fioricet, eletriptan, prednisone, hydrochloroquine, lansoprazole, ranitidine, phenobarbital, and polyethylene glycol.

In the ED she was ill-appearing, uncomfortable, and actively complaining of abdominal pain. Her vital signs were as follows: blood pressure 123/93, heart rate 126 beats/min, temperature $37.6^{\circ} \mathrm{C}$, respiratory rate of $24 / \mathrm{min}$, and pulse oximetry of $100 \%$ on room air. Her physical exam showed that her lung sounds were clear to auscultation and the heart rate was sinus tachycardia with regular rhythm without murmur on auscultation. Her abdomen was distended and diffusely tender without guarding or rebound.

Initial laboratory values were as follows: white blood cell count 10,000 cells/uL (normal $4.5-13 \times 10^{3} / \mathrm{uL}$ ) with $78.5 \%$ 


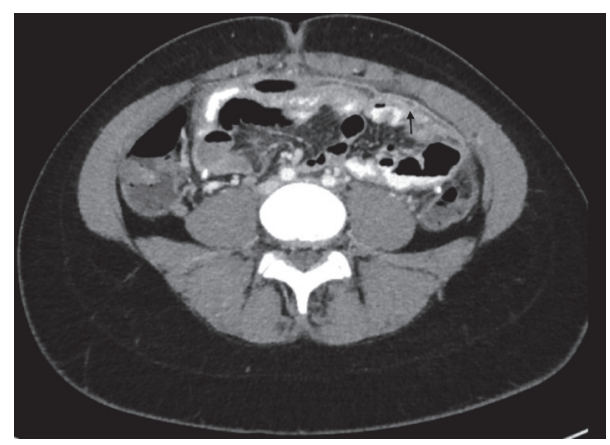

FIgURE 1: Peritonitis due to NVS. Abdominal CT scan with contrast. The arrow points to the hyperintensity demonstrating fluid accumulation consistent with peritonitis.

neutrophils (normal 34-64\%), 11.3\% lymphocytes (normal 35-45\%), 0.2\% eosinophils (normal 0-3\%), hemoglobin concentration $10.8 \mathrm{~g} / \mathrm{dL}$ (normal $12-16 \mathrm{~g} / \mathrm{dL}$ ), platelets $289,000 / \mathrm{uL}$ (normal $150-450 \times 10^{3} / \mathrm{uL}$ ), C-reactive protein (CRP) $25.2 \mathrm{mg} / \mathrm{dL}$ (normal $<1.0 \mathrm{mg} / \mathrm{dL}$ ), and gammaglutamyl transferase $349 \mathrm{U} / \mathrm{L}$ (normal range 7-32 U/L). Alanine aminotransferase and aspartate aminotransferase values along with amylase and lipase levels were within normal limits. A CT scan of the head with and without contrast showed stable appearance of shunted hydrocephalus. An abdominal CT scan performed with oral and intravenous contrast showed moderate pelvic-free fluid with peritoneal enhancement and mild to moderate diffuse thickening of the adjacent ileum and terminal ileum (Figure 1). The two ventriculoperitoneal shunt tubes were observed with no evidence of loculated fluid around the tip of either shunt.

The presence of abdominal pain, significantly elevated CRP, and accumulation of peritoneal fluid on CT scan were consistent with acute peritonitis and she was admitted to the Pediatric Intensive Care Unit for further care. She underwent laparoscopic surgery and VP shunt externalization within a few hours of admission where peritoneal cavity and cerebral spinal fluid (CSF) were collected for bacterial culture. A blood culture was not obtained at admission as she was afebrile with a normal white blood cell count and NVS was not initially suspected as pathogen. Empiric antibiotic therapy was started with vancomycin, piperacillin/tazobactam, and metronidazole. The Gram stain of both the CSF and peritoneal fluid showed Gram positive cocci. Despite antibiotic therapy, she developed fevers as high as $39.1^{\circ} \mathrm{C}$ on the third day of admission.

Both CSF and peritoneal cultures were positive on day 3 with faint bacterial growth on the chocolate agar plates. Growth on blood agar plates occurred only in the presence of $\beta$-hemolytic Staphylococcus aureus (Figure 2) and the organism was identified as NVS species. All specimens from the peritoneum and CSF grew pure NVS without other bacteria. The laboratory was unable to determine the antibiotic susceptibilities as it could not recover viable organism under conditions that would permit routine susceptibility testing or further identification of the organism at the genus level.

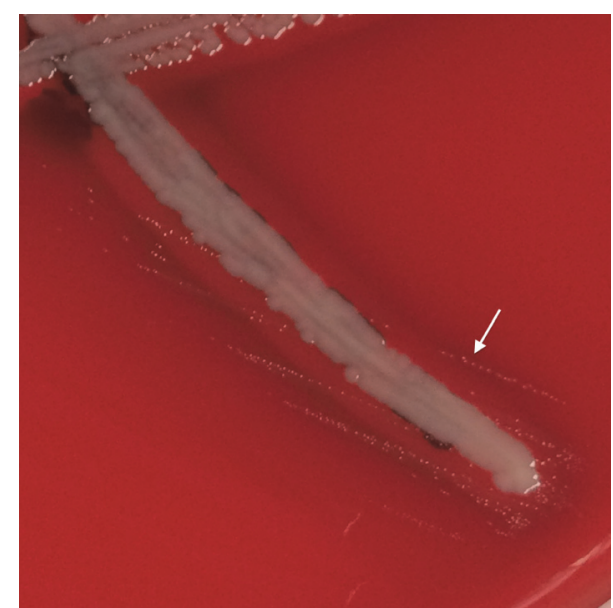

FIGURE 2: Satellite growth of nutritional variant streptococci (NVS) around Staphylococcus aureus. Small colonies of NVS are seen in areas on the blood agar plate where $S$. aureus has completely lysed red blood cells ( $\beta$-hemolysis), releasing nutrients required for NVS growth.

Based on the culture results, rifampin was added $300 \mathrm{mg}$ every 12 hours (adult dose) and the vancomycin was continued $(600 \mathrm{mg}(15 \mathrm{mg} / \mathrm{kg} / \mathrm{dose})$ every 8 hours to maintain a trough of $15-20 \mathrm{mg} / \mathrm{L}$ ). Piperacillin/tazobactam was discontinued but the primary team remained concerned about anaerobic bacteria not recovered via standard culture techniques so they continued metronidazole ( $400 \mathrm{mg}$ every 6 hours $(40 \mathrm{mg} / \mathrm{kg} /$ day)). CSF cultures collected on a daily basis for the next 7 days after the initial positive cultures remained with no growth demonstrating a rapid clearance of her CNS infection. She defervesced, her abdominal pain improved, and the recommendation was made to convert her shunt system to a ventriculoatrial (VA) shunt 2-3 weeks after sterilization of the central nervous system. A blood culture taken on approximately day 12 of hospitalization was negative prior to shunt revision and she had three additional negative blood cultures during antibiotic therapy. She was treated with rifampin for a total of 11 days and originally scheduled for four weeks of vancomycin therapy but this was extended to 12 weeks until her CRP normalized which would be a sufficient length of therapy for endocarditis. However, she continued to have no murmur on cardiac auscultation and an echocardiogram was not performed. One year later she still has the VA-shunt in place and has not had any subsequent positive blood cultures, documented peritonitis, CNS infection, or signs and symptoms of clinical relapse.

\section{Discussion}

There is great variety in the types of Gram positive and Gram negative organisms that can cause peritonitis. In the presented case, the Gram positive organisms seen on staining of the CSF and peritoneal fluid grew poorly in the clinical microbiology laboratory, suggesting this was not a common pathogen. Satellite growth in the presence of $S$. aureus confirmed a nutritionally fastidious organism (Figure 2). In fact, NVS should be considered when the Gram stain of 
the clinical specimen reveals Gram positive cocci in chains consistent with streptococci that grow poorly or not at all on routine culture. These bacteria do not grow well on agar media apart from hematin and grow best with the addition of PHC pyridoxal hydrochloride which provides the bacteria with vitamin B6 necessary for their growth [2].

There is one report of peritonitis in an adult patient with end stage renal disease where the peritoneal cultures, like this case, grew pure NVS (Abiotrophia sp.) [5]. The majority of NVS of the CNS are associated with either endocarditis, or a recent neurosurgical procedure including one case of a patient with recent VP shunt revision [5-8]. By the time this organism was identified in this patient she was on antibiotic therapy and improving so an echocardiogram was not performed and her infection was attributed to her recent VP shunt revision. However, these previously reported CNS infections have not been associated with peritonitis. Interestingly, this patient's last shunt revision was done less than two months prior to presentation.

Very little is known about the microbial pathogenesis of NVS. Okada et al. studied 17 Abiotrophia strains from the oral cavity of healthy patients and seven from patients with proven endocarditis in a rat model of endocarditis [9]. All seven Abiotrophia strains that caused endocarditis in people were highly infective in the rat model. Of the strains from healthy controls, the infectivity appeared to be species dependent with $A$. adiacens showing the highest rates of infectivity and $A$. para-adiacens and A. elegans the lowest [9]. Interestingly, the highly infective $A$. adiacens strains tended to show markedly higher fibronectin-binding capacity compared with less infective strains, suggesting a possible relationship between the fibronectin-binding capacity and ability to cause disease [9]. In this case, one possibility is that the NVS attached to and colonized the VP tubing in the subsequent weeks after the surgical revision and this contributed to CSF infection that seeded the peritoneal cavity resulting in peritonitis.

The Infective Endocarditis treatment guidelines recommend aggressive treatment with either a penicillin (i.e., intravenous ampicillin or aqueous crystalline penicillin $G$ sodium) and intravenous gentamicin or the use of intravenous vancomycin and gentamicin [10]. There are no Clinical Laboratory Standard Institute guidelines for antibiotic susceptibility testing for NVS so the ideal antimicrobial regimen is unclear with at least one report of Abiotrophia with a vancomycin minimum inhibitory concentration (MIC) of 2-3 mg/L. [5] Further, the endocarditis guidelines state that even if susceptibility is determined, it may be inaccurate and not correlate with clinical cure [11]. Literature reports of small case series show that most strains are thought to be susceptible to vancomycin, gentamicin, and rifampin and that combination therapy may be advisable to prevent relapse $[2,5]$. In case series and reports of NVS infections of the CNS, the patients were treated with a beta-lactam or vancomycin as primary therapy with gentamicin and/or rifampin added on for synergy [5-7]. The choice of these agents is consistent with recommendations in the Infective Endocarditis guidelines where systemic concentrations are easy to achieve, but this does not guarantee disease eradication $[10,11]$.
In cases of CNS infection such as this, appropriate penetration of antibiotics into the CSF at therapeutic levels is essential. Therefore, vancomycin was dosed aggressively to achieve trough concentrations of $15-20 \mathrm{mg} / \mathrm{L}$. Further, rifampin rather than gentamicin was employed because intravenous gentamicin has not demonstrated detectible CNS concentrations beyond the neonatal period [12]. Instead, rifampin with approximately $10 \%$ CNS penetration was used to provide synergistic activity. However, no appropriately powered clinical trial has compared various treatment options to determine optimal therapy so clinical response and careful follow-up are currently the best way to determine if the chosen antibiotic regimen is working.

\section{Consent}

After consultation with the pediatric residency research coordinator, verbal informed consent for this case report was obtained from the patient's mother over the phone by Sussi Vivar in the presence of a witness and documented.

\section{Competing Interests}

The authors declare that they have no competing interests.

\section{References}

[1] J. J. Christensen and R. R. Facklam, "Granulicatella and Abiotrophia species from human clinical specimens," Journal of Clinical Microbiology, vol. 39, no. 10, pp. 3520-3523, 2001.

[2] K. L. Ruoff, "Nutritionally variant streptococci," Clinical Microbiology Reviews, vol. 4, no. 2, pp. 184-190, 1991.

[3] D. P. Bhat, L. Nagaraju, B. I. Asmar, and S. Aggarwal, "Abiotrophia endocarditis in children with no underlying heart disease: a rare but a virulent organism," Congenital Heart Disease, vol. 9, no. 4, pp. E116-E120, 2014.

[4] E. Cerceo, J. D. Christie, I. Nachamkin, and E. Lautenbach, "Central nervous system infections due to Abiotrophia and Granulicatella species: an emerging challenge?" Diagnostic Microbiology and Infectious Disease, vol. 48, no. 3, pp. 161-165, 2004.

[5] U. Arslan, I. Guney, S. Yuksekkaya, H. Atalay, and H. Turk Dagý, "First case of peritonitis due to Ablotrophia defectiva [9]," Peritoneal Dialysis International, vol. 26, no. 6, pp. 725-726, 2006.

[6] D. D. Kohok, A. Parashar, V. Punnam, and A. Tandar, "Subarachnoid hemorrhage in a patient with abiotrophia defectiva endocarditis," American Journal of the Medical Sciences, vol. 341, no. 2, pp. 157-159, 2011.

[7] F. Flor-De-Lima, L. Lisboa, A. Sarmento, J. Almeida, and T. Mota, "Mycotic brain aneurysm and cerebral hemorrhagic stroke: a pediatric case report," European Journal of Pediatrics, vol. 172, no. 9, pp. 1285-1286, 2013.

[8] D. Tena, S. Solís, S. Lainez et al., "Meningitis caused by abiotrophia defectiva: case report and literature review," Infection, vol. 41, no. 2, pp. 571-574, 2013.

[9] Y. Okada, K. Kitada, M. Takagaki, H.-O. Ito, and M. Inoue, "Endocardiac infectivity and binding to extracellular matrix proteins of oral Abiotrophia species," FEMS Immunology and Medical Microbiology, vol. 27, no. 3, pp. 257-261, 2000. 
[10] R. S. Baltimore, M. Gewitz, L. M. Baddour et al., "Infective endocarditis in childhood: 2015 update: a scientific statement from the American Heart Association," Circulation, vol. 132, no. 15, pp. 1487-1515, 2015.

[11] L. M. Baddour, W. R. Wilson, A. S. Bayer et al., "Infective endocarditis: diagnosis, antimicrobial therapy, and management of complications a statement for healthcare professionals from the Committee on Rheumatic Fever, Endocarditis, and Kawasaki Disease, Council on Cardiovascular Disease in the Young, and the Councils on Clinical Cardiology, Stroke, and Cardiovascular Surgery and Anesthesia, American Heart Associationexecutive summary," Circulation, vol. 111, no. 23, pp. 3167-3184, 2005.

[12] A. K. Sullins and S. M. Abdel-Rahman, "Pharmacokinetics of antibacterial agents in the CSF of children and adolescents," Pediatric Drugs, vol. 15, no. 2, pp. 93-117, 2013. 


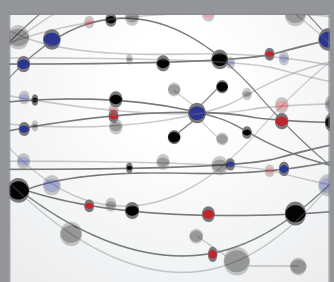

The Scientific World Journal
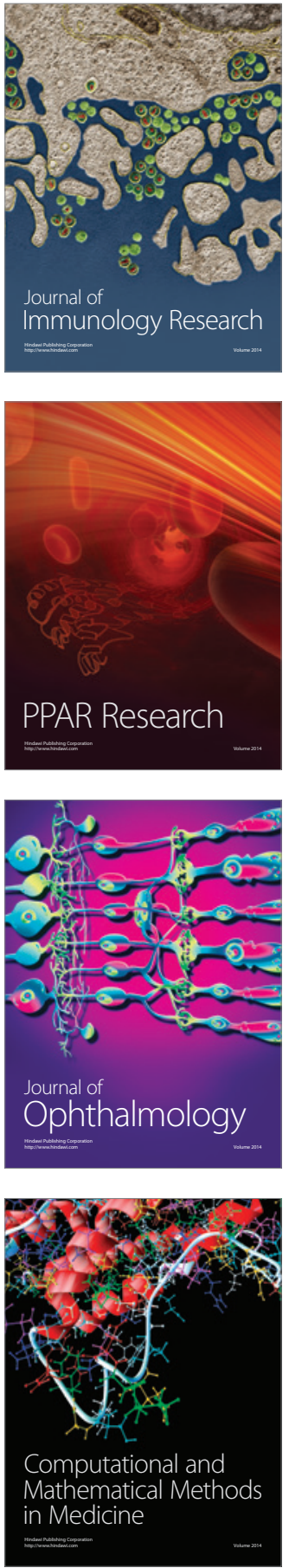

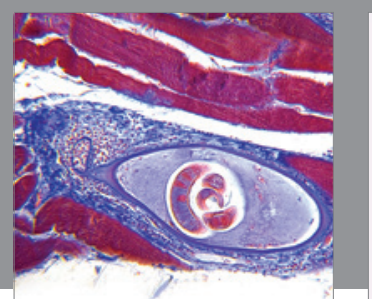

Gastroenterology Research and Practice
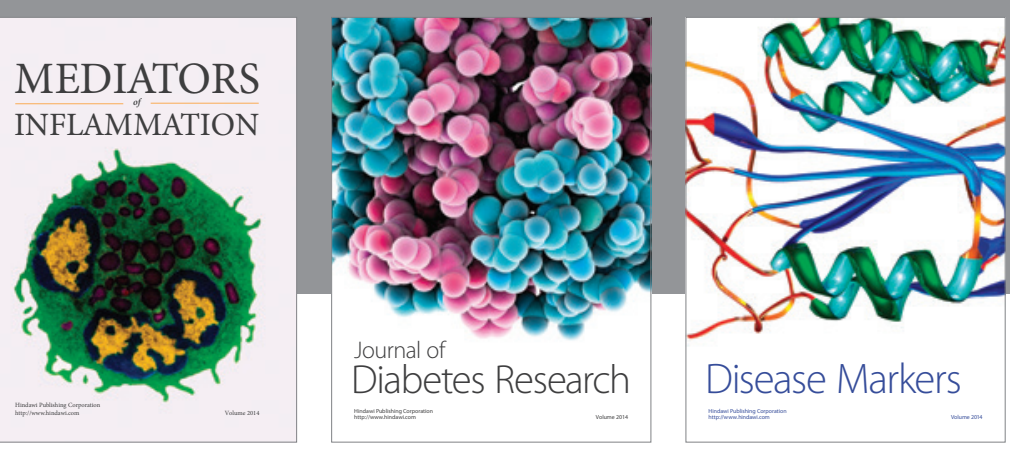

Disease Markers

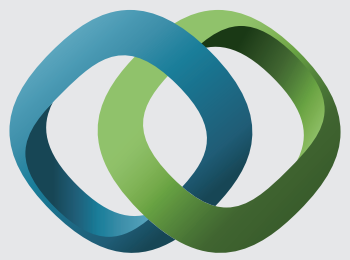

\section{Hindawi}

Submit your manuscripts at

https://www.hindawi.com
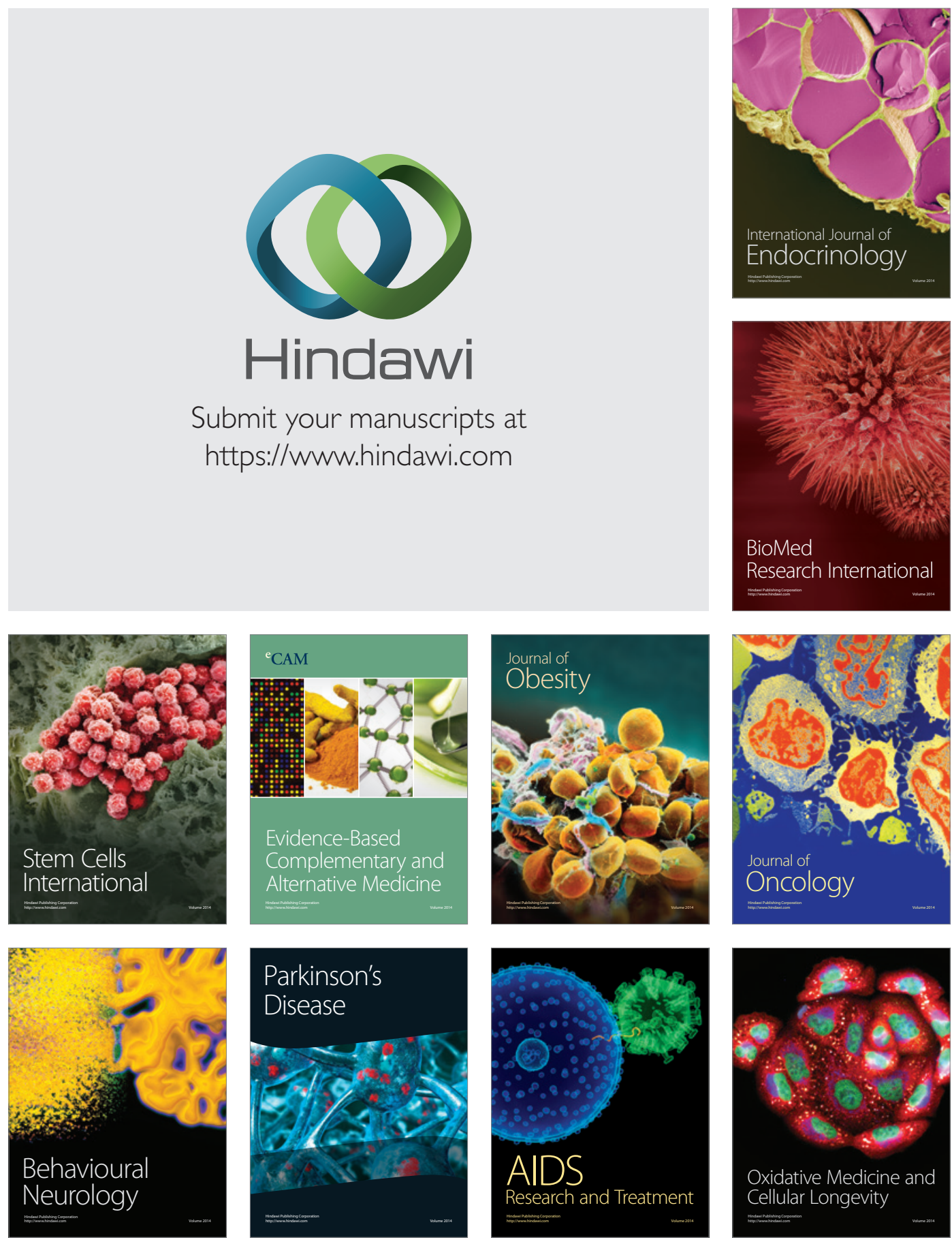